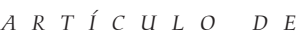
I N V ES TI G A C I O N C I E $N$ N T I I F I C C A

TE C N O L Ó G I C A

\section{FACTORES QUE AFECTAN LA IMPLEMENTACIÓN DE INNOVACIONES EN EL AULA DE CLASE DE NIVEL BÁSICO EN COLOMBIA}

\author{
ASPECTS THAT AFFECT THE IMPLEMENTATION OF INNOVATIONS \\ IN MIDDLE SCHOOL CLASSROOM IN COLOMBIA
}

Por: Juan Habib Bendeck Soto*

*Coordinador de Área Profesional. Corporación Universitaria Americana. Facultad de Ingeniería de Sistemas. Medellín, Antioquia (Colombia). E-mail: jhbendeck@gmail.com

- jendeck@coruniame-jbendeck@coruniamericana.edu.co. Teléfono: +573184936263 .

\author{
Por. Juan Habib Bendeck Soto
}

Recibido: 28 de octubre de 2015 - Aprobado: 14 de julio de 2016

\section{RESUMEN}

Se realizó una investigación de cómo se implementan las innovaciones en el aula, en el sector de Educación Básica y Media, en la que se tomó como objetivo central identificar y analizar los diversos factores que influyen tanto de manera positiva como negativa en las innovaciones educativas implementadas por los profesores en el aula escolar, para sistematizar y difundir dichas experiencias en la comunidad educativa, de manera que se demostrara el impacto que tiene aplicar estas propuestas; para esto se trabajó bajo la metodología de tipo cualitativa, y se emplearon las observaciones de clase, las entrevistas semi-estructuradas y la revisión de literatura especializada como técnicas de recolección de datos, y triangulación metodológica, para marcar los resultados obtenidos y organizarlos de manera detallada, con el fin de dar a conocer ciertos factores que influyen en la aplicación de estas propuestas, apoyadas en parte con el modelo de Aprendizaje por Proyectos. Para las entrevistas y observaciones se tomó una muestra de 8 estudiantes, 3 docentes y 1 directivo docente, de la población de dicha institución. Los resultados obtenidos mostraron que: 1. Este proceso requiere de mucho acompañamiento de parte de las directivas para que pueda tener éxito, 2. Se requiere del apoyo de otras áreas para que la innovación tenga el impacto deseado en los estudiantes, 3. Se debe motivar a los estudiantes para participar de estas actividades, y más importante aún, dejarles en claro el propósito de dicha innovación y el beneficio que obtendrán de ella, y 4. Indagar cómo se registran o sistematizan estas experiencias, en pro de realizar los cambios adecuados para que sean un éxito en el aula y puedan ser promovidas dentro de la institución educativa.

Palabras claves: ABP, co-responsabilidad, educación básica, enseñanza, factores, impacto, implementación, innovación educativa, praxis pedagógica.

\begin{abstract}
A research on how to implement educational innovations in a regular classroom of Elementary and Middle School was conducted, in which the key objective was to identify and analyze the different factors that impact in a positive and negative way on the innovations applied by teachers from these sections, to systematize and expand these experiences in an educational community that the impact of these propositions could be proven. This work was conducted by using semi-structured interviews and with the revision of specialized literature as data collection techniques; as well as the methodological triangulation
\end{abstract}


to mark the obtained results and organize them in a detailed way, with the purpose of presenting some factors that affect these proposals, supported by the PBL (Project-Based Learning methodology). For the implementation of interviews and observations, it was taken a sample of eight (8) students, three (3) teachers and one (1) school directive, from the overall population of such institution. The obtained results evidenced that 1 . This process requires the companion of the directive school personnel for it to be successful; 2. The support from other academic areas is required so that the innovation can have the expected impact in the students; 3. Students must be motivated in order to participate in these activities, and mostly, make them understand the key purpose of such innovation and the academic benefits they will get from it; and 4. Inquire on how to register or systematize these experiences, leading toward the proper changes to make them be successful in the classroom and that they can be promoted within the school.

Keywords: ABP, co-responsibility, basic education, teaching, factors, impact, implementation, educational innovation, pedagogical praxis.

\section{Introducción}

n esta investigación se buscó indagar sobre la praxis pedagógica de $\checkmark$ los profesores, para conocer las innovaciones que realizan en el aula, particularmente aquellas que el colegio ha promovido en cuanto a la implementación de metodologías de aprendizaje y uso de TIC; Carbajo (1997) describe el proceso de innovar en el aula como la idea de utilización de materiales y modos de hacer radicalmente distintos de los ya existentes, por lo que se aplica dentro del modelo educativo de la institución orientado a la enseñanza para la comprensión y al Aprendizaje Basado en Proyectos (ABP), por lo que se propuso la siguiente pregunta general: ¿Qué factores limitan la implementación de innovaciones en el salón de clase desde la perspectiva docente?

Durante el desarrollo de esta investigación, se le permitió al docente reflexionar sobre un nuevo escenario con innovaciones funcionales, en el que asumieron un papel reflexivo sobre lo que hacen a diario en su ejercicio y en la institución, y establecieron compromisos para implementar los cambios que se requieren tanto para ellos como para los alumnos en aras de producir un conocimiento y propagar estos cambios en otros contextos (Hernández y Castañeda, 2001). Además, fue desarrollada en relación a que los docentes son quienes están en constante generación de ideas y siempre están buscando cambios que mejoren la calidad educativa; por ende, tales ideas tendrán coherencia con el sistema que propone la institución y permita aportar soluciones a problemas inmediatos (Díaz, Díez, San Fabián y Martínez, 1996.)

Por esta razón, toda la información relevante recolectada durante esta propuesta estuvo basada en la conceptualización de las innovaciones desde la 
óptica del profesor para propiciar la reflexión sobre su práctica pedagógica y demostrar el cambio requerido en esta era del conocimiento. A pesar de estas situaciones, es válido resaltar que las innovaciones que realizan los profesores en su contexto escolar no tiene el mismo significado o el mismo valor para todos si no están orientados a una producción de conocimiento específico y con valor para la vida en sociedad; los docentes reconocen y consideran que una clave para generar un cambio innovador debe tomar como punto de partida cuestionar sus prácticas en el aula, reconocer los contenidos que sirven y no sirven, los cambios viables y los no viables, hacer autocrítica sobre la forma en cómo está realizando su trabajo con los estudiantes y garantizar que sus innovaciones mejoraran las situaciones anteriores.

\section{Materiales y método}

1 método de investigación aplicado para este proyecto fue cualitativo, con un enfoque fenomenológico y etnográfico, buscando obtener respuestas a las preguntas de investigación formuladas sobre la innovación educativa en una institución de educación básica, la cual fue llevada a cabo de febrero a noviembre de 2014, en un colegio particular de la ciudad de Medellín, Colombia, en la que se tomó como contexto las vivencias de los estudiantes y la práctica docente en el aula, frente a la implementación de proyectos de innovación educativa, conocer sus ideas y cómo son las innovaciones educativas implementadas en el aula, partiendo desde un punto de vista fenomenológico para conocer las experiencias vividas por ellos con respecto a la aplicación de estas nuevas metodologías de enseñanza y aprendizaje (Valenzuela y Flores, 2011).

Para llevar a cabo esto, se realizó la aplicación de entrevistas semi-estructuradas con base al objetivo general trazado para la investigación, la cual dio respuesta al planteamiento de problema que se creó. Dicho instrumento se le aplicó a 3 docentes, 8 estudiantes y un directivo, los cuales se eligieron por su participación en procesos innovadores de la institución y por su experiencia en dinamizar las actividades de sus respectivas áreas.

Complementando a estas entrevistas, se realizó observación no participante para este proyecto de investigación, en la que el investigador estuvo presente en las clases pero no interactuó con estudiantes y docentes. Esto implicó acudir a observar las clases de los diferentes docentes que se incluyeron en el proyecto junto con las planeaciones de cada clase, observar cómo impartían su clase, qué factores y qué recursos utilizaban para dinamizar la enseñanza de 
sus respectivas áreas, el impacto que tenía dicha actividad en los estudiantes; siempre tomando notas de campo organizadas y estructuradas para facilitar luego la descripción e interpretación. Para esto, se generó un registro de observaciones para recolectar datos en el aula, lo que permitió realizar un análisis más a fondo de las diferentes metodologías aplicadas y observarlas de manera directa. Se diseñó una matriz de observación considerando los objetivos generales y específicos de la investigación, y tomar como referencias las planeaciones de clase, utilizando técnicas de revisión documental.

Además, se hizo la revisión de los diversos currículos manejados por los docentes que hacen parte de la investigación, para revisar la misión, la visión, valores institucionales y los Proyectos Educativos Institucionales (PEI), en cuanto a la aplicación de métodos innovadores en el aula para la formación académica y personal de las estudiantes. Para concertar las citas y efectuar las entrevistas, se les pidió tanto a estudiantes como al directivo docente de acudir a la sala de computadores de la institución para ser entrevistados en un horario específico. Se les explicó en qué consistía dicho instrumento y cuál era el propósito de la investigación. Para observar las clases se estableció con los profesores un calendario de visitas.

Para aplicar estos instrumentos, se hizo una solicitud a la dirección de estudios de la institución, con el fin de que dicha propuesta sea aplicada y se recolectaran datos útiles para el personal docente; además, se contó con la autorización de los docentes y directivos participantes. Luego, se aplicó una prueba piloto a la entrevista y al registro de observación. Las personas que se seleccionaron para hacer la prueba piloto de los instrumentos fueron seleccionados de forma aleatoria y se codificaron de la siguiente manera: JB: Docente de Tecnología; MPM: Docente de Ciencias Sociales; MSD: Docente de Lengua Castellana; EH6: Estudiante 1 de $6^{\circ}$ Grado; SH6: Estudiante 2 de $6^{\circ}$ Grado; NC7: Estudiante 1 de $7^{\circ}$ Grado; LC7: Estudiante 2 de $7^{\circ}$ Grado; AL8: Estudiante 1 de 80; AR8: Estudiante 2 de $8^{\circ}$ Grado; SL9: Estudiante 1 de $9^{\circ}$ Grado; JA9: Estudiante 2 de $9^{\circ}$ Grado; y CG: Directivo.

\section{Resultados y discusión}

Y on base a los resultados obtenidos de esta investigación, se determinaron las siguientes categorías con respecto a la innovación educativa en el aula: Factores que limitan la aplicación (Tiempo, motivación, participación docente), Aprendizaje Basado en Proyectos y Sistematización de la innovación. Estos se describen en la siguiente tabla 1., junto con su definición: 


\begin{tabular}{|l|l|}
\hline Categorías & Definición \\
\hline Tiempo & $\begin{array}{l}\text { Elemento que puede impedir que la innovación se lleve a cabo en } \\
\text { un espacio temporal, debido al horario escolar o a las actividades } \\
\text { curriculares de la institución. }\end{array}$ \\
\hline Motivación & $\begin{array}{l}\text { Componente esencial de parte del estudiante o docente, para aceptar } \\
\text { o rechazar la innovación }\end{array}$ \\
\hline Participación docente & $\begin{array}{l}\text { Compromiso de los docentes para su integración con la propuesta } \\
\text { de innovación determinada }\end{array}$ \\
\hline
\end{tabular}

Tabla 1. Factores que limitan la aplicación de innovaciones en el aula, detectados dentro de la investigación

Dentro del marco de la innovación educativa, es importante tener en cuenta que una actividad o propuesta para ser aplicada en el aula debe tener ciertos factores que determinen de cierta forma qué componentes físicos, qué fundamentos teóricos, qué actores deben intervenir en esto, y el conocimiento que se desea que los alumnos aprendan significativamente, esto debido a que el conocimiento se convierte en elemento clave transversal con la información y la innovación, ejes relevantes de este nuevo sistema globalizado (Villa Lever, 2014); no obstante, siempre existen aspectos que influyen negativamente para que pueda tener éxito en su implementación, ya que al no contar con las herramientas tecnológicas, el desinterés por realizar las diferentes actividades de la propuesta, la ausencia de disciplina en el aula, o las características del lugar de trabajo, son algunos de los aspectos observados durante la investigación, los cuales impacta la aplicación de innovaciones en el aula.

El Ministerio de Educación de España (2011) resalta los siguientes requisitos para elaborar un proyecto de innovación:

- Contar con los recursos didácticos necesarios.

- Distribuir las tareas y cumplir con las mismas.

- Asignar miembros coordinadores dentro del proyecto, que garanticen su cumplimiento.

- Establecer un funcionamiento interno basado en normas y reglas de las tareas a realizar y cómo se van a realizar.

- Contar con espacios adecuados.

- Distribuir el tiempo de forma óptima y productiva.

- Realizar una evaluación continua.

Cuando uno de estos elementos no se encuentra presente, es donde los actores educativos tienen dificultades para participar e intervenir en la propuesta 
innovadora. Es por esto que, en esta investigación se detectaron tres limitantes en la institución al momento de realizar una actividad educativa de carácter innovador: el tiempo, la motivación y la participación docente.

Tiempo: Ríos (2010) anota que, uno de los factores más importantes, que limita mucho la implementación de una innovación, es la falta de tiempo de los docentes, debido a la excesiva carga laboral, la cual les dificulta cumplir las tareas asignadas dentro del cronograma de actividades escolares, recorta considerablemente el tiempo de aplicación y puede descuadrar el horario establecido por parte del docente para aplicarla.

Los tres docentes entrevistados, básicamente coincidieron con esta premisa en cuanto al factor del tiempo, dado que muchas veces los proyectos innovadores que se aplican en el aula propuesta pueden requerir varios bloques (horas de clase) para su mejor desarrollo y para que produzca mejores resultados en aras de conseguir los objetivos trazados al inicio de la propuesta; JB manifestó presentar dificultades con el tiempo: "El factor del tiempo es clave, dado que no se dispone de mucho tiempo y el que se tiene a la mano, muchas veces se destina a actividades institucionales programadas dentro del horario escolar"; y es que, cuando este factor influye de manera negativa en la implementación de una innovación, se puede reflejar un avance muy lento, hasta el punto en que pueda provocar no solo que la propuesta desaparezca o que el logro de objetivos no se concrete, sino el desinterés de parte de los estudiantes, así como la desmotivación del docente para proponer otra idea en el aula.

Otro punto importante a considerar es la distribución del tiempo en las actividades que desarrollan los estudiantes. Se reconoce que los docentes poseen conocimientos pedagógicos generales, relacionado con las estrategias de enseñanza aplicadas en clase, de igual manera se tiene en cuenta el tiempo que será invertido en las actividades y el nivel de aprendizaje que espera de cada uno de sus estudiantes (Marcelo, 2013), así como el tiempo de espera que debe llevar con aquellos que presenten Necesidades Educativas Especiales (NEE), para poder obtener un resultado mucho más general de su propuesta innovadora.

Se pudo observar durante el desarrollo de los proyectos, situaciones como la que presentó JA9, quien manifestó que "no se le ha dado el tiempo suficiente y las otras compañeras también asienten sobre esto"; por eso, es importante el hecho de que el docente sea claro en las instrucciones y distribuya el tiempo 
de trabajo, que regule la atención de los estudiantes de manera que no se distraigan. Con relación a este comentario, el docente les aclaró que "han tenido dos clases para terminar esto y que deberían estar ya en la fase dos del proyecto, en la semana actual", principalmente porque se vio a muchas estudiantes haciendo cosas diferentes al trabajo planteado a desarrollar, y no empleando el tiempo requerido para realizar la actividad.

Motivación: Pintrich (1994) afirma que la motivación académica es sinónima de realizar un buen trabajo en el aula, y eso se refleja en las conductas que llevan a cabo los estudiantes dentro del aula al momento de la innovación. Dicho momento conlleva a que se den interacciones entre tres componentes: el contexto en el que se da la clase, los sentimientos y creencias de los estudiantes, y los comportamientos a revisar de los estudiantes.

Es aquí donde la motivación de los actores (docente y estudiante) influye en la ejecución de estas propuestas, hasta el punto de llegar a ser uno de los principales objetivos de los profesores: motivar a sus estudiantes. Una prueba de ello la daba JB, al argumentar que "cuando mis estudiantes reciben una propuesta innovadora, llegan motivadas y a la expectativa de las diversas actividades propuestas, mostrando su interés para trabajar en clase". Esto se observó en el trabajo que realizaron las estudiantes durante la clase, en la que, el docente realizó el comentario para incentivar la motivación para iniciar el proyecto, y de inmediato se vio la reacción de las niñas que regularmente manifiestan apatía y desinterés por los proyectos; el docente, de forma serena, les planteó las condiciones y los requerimientos para desarrollar durante todo un período (dos meses y medio), y ahí fue donde las estudiantes calmaron los ánimos y prestaron más atención a lo dicho en la clase.

Cuando se realizó la primera observación de clase de español, cuando MSD llevó a las estudiantes a las salas de cómputo, lo primero que hicieron fue acceder a sitios como Facebook o a YouTube, sin atender las instrucciones previamente dichas; esto es un común denominador en las niñas de esta era, lo que demuestra que, cuando la propuesta no es de su interés, pueden simplemente aislarse en otras cosas y no atender con seriedad y compromiso la propuesta del docente. En el caso de los estudiantes, Ríos (2003) menciona que, muchas veces pueden manifestar temor a metodologías desconocidas, otro es la resistencia al cambio y las mejoras al interior del aula, o también por la poca experiencia al trabajar con herramientas TIC de las cuales no tienen conocimiento alguno. 
Cabe resaltar que en una de las entrevistas realizada a AR8, ella comentó que "generalmente si se utilizan las TIC en el aula, y ayuda a cambiar el método de enseñanza al que estamos acostumbradas; aunque muchas veces utilizamos el mismo método tantas veces que el concepto de innovación en la clase se pierde", lo que conlleva a pensar que el uso constante de una herramienta se convierte en una rutina y se pierde el interés de los estudiantes, e incluso, perder todo el foco de aprendizaje en la asignatura respectiva, por lo que los docentes deben ser muy cuidadosos al momento de proponer estas actividades y no convertirlas en un ejercicio diario y sin sentido alguno. Por consiguiente, es necesario que los docentes tomen su tiempo, analizar las temáticas del currículo detalladamente, y planear dichas actividades teniendo en cuenta los componentes pedagógicos claves a evaluar en los estudiantes (trabajo colaborativo, metodología de trabajo), la intensidad de trabajo con la cual trabajarán y la cantidad de personas y recursos involucradas en la propuesta (Rimari, 2002).

Para Polanco (2005), la motivación es tan influyente en el aprendizaje que termina convirtiéndose en el principal objetivo en el aula, y lo ideal es que, dentro de una propuesta de innovación, el docente proponga una motivación de tipo intrínseca, es decir, que los motive a vivir la propuesta más allá del logro cognitivo a alcanzar, y que les despierte pasión por la(s) asignatura(s) que promueven dicha actividad. Sin embargo, cuando el docente determina que dicha actividad tiene un valor cognitivo, las estudiantes automáticamente cambian el modo de trabajo de manera forzada, sintiendo presión para entregar un producto y tener que cumplir con eso, ya que de lo contrario pueden tener un mal resultado académico; una de las estudiantes entrevistadas, NC7, comentaba "me motivan diciéndome que vale 30\% de la materia; a veces ponen temas a los cuales a uno le gustan más y le parecen más interesantes, ahí es cuando uno se motiva más".

Un detalle importante, el cual es muy común en los estudiantes de la generación actual, es la manera en cómo el docente presente esta propuesta a los estudiantes. Si el docente introduce este tipo de propuestas de manera plana, sin mostrar a los estudiantes la importancia, la dinámica de las actividades a desarrollar y la relevancia del conocimiento que aprenderán, provocará que los estudiantes vean todo como algo rutinario, simple y sin interés alguno de participar; en este escenario, el docente automáticamente perderá toda intención de ganar terreno frente a los estudiantes en el aula y generará desmotivación automática, aun si incorporara uso de herramientas tecnológicas o planeara actividades lúdicas con un propósito definido. 
Participación docente: Complementando a esta motivación, otro factor que también afecta la implementación de innovaciones en una institución es la falta de participación de los docentes, ya sea por descontento, cansancio debido a la complejidad de los nuevos roles y tareas, por la naturaleza cambiante del conocimiento y la educación que han de desarrollar en unas condiciones difíciles y/o desconocidas. Todo ello conlleva a conductas muy pesimistas en el profesorado que no le interesa saber nada de innovaciones (Carbonell, 2012).

JB manifestó, con respecto a la transversalidad y el apoyo de otros docentes para participar en proyectos innovadores, que "todo depende de la actividad o proyecto propuesto para realizar una propuesta transversal y trabajar entre otras áreas, bien sea proporcionando el tiempo y el espacio para trabajar en dicha actividad"; esto demuestra que, muchos docentes se preocupan por cumplir a cabalidad lo propuesto en sus planeaciones de clase y pueden permitir ajustes para aplicarlas en conjunto con otras áreas. Sin embargo, si cada profesor propone incluir metodologías con un número de actividades y lo realiza por iniciativa propia, existe un peligro de que competencias transversales de importancia no se desarrollen, y esta parte es relevante en el momento de aplicarla, dado que el individualismo constituye la versión más negativa del aislamiento, la libertad de cátedra o conciencia y la autonomía, en contraposición con la individualidad que se relaciona con la formación de un criterio y juicio propio y con el trabajo independiente y autónomo susceptible de confluir en el colectivo y enriquecerlo (Carbonell, 2012).

Por otro lado, comentarios como el expresado por MPM: "Encuentro limitaciones, como el acceso a cierta información puesto que esta se encuentra en un idioma diferente al que debo enseñar en clase"; es una muestra clara que, si su asignatura es enseñada en inglés, y debe enseñar por ejemplo, historia de Colombia, por cuestiones administrativas, es básicamente enseñar dos currículos diferentes, lo que para esta docente implicaría traducir todos los textos existentes sobre la historia de Colombia al inglés, lo que significa una desmotivación muy importante al momento de que quisiera aplicar alguna actividad innovadora dentro del aula; esto es, la influencia de las directivas docentes en las temáticas propuestas por manejar currículos planos y estandarizados no le da al docente la libertad necesaria para planear actividades que puedan darle al estudiante la capacidad de aprender de manera dinámica e interactiva una temática específica.

Y por último, la no participación de algunos docentes en las propuestas de investigación también se expresa en las respuestas de los docentes, 
bien sea porque no tienen cabida dentro de los parámetros definidos por los directivos para las actividades o debido a la negligencia y resistencia a participar por el no seguimiento del currículo que ya tienen definido en su área respectiva. Este factor es uno de los más llamativos, dado que este tipo de docentes solo se limitan a "dictar catedra" en un aula de clases de educación básica sin tener presente el proceso de desarrollo de habilidades y formación en valores del estudiante, puesto que para este tipo de estudiantes, es clave el entendimiento del conocimiento que están recibiendo y del saber aplicarlo en diversos contextos; esta situación, por lo general, tiende a que sean docentes perezosos sin interés alguno en innovar, y recurrir a la típica clase tradicional, método pedagógico poco convencional para esta generación de estudiantes.

Cuando se les preguntó a los docentes sobre el liderazgo y aplicación de los proyectos, y la participación de sus compañeros, comentaron que "cuando la innovación está relacionada con mi área de conocimiento, las directivas me solicitan que la aplique y la lidere. En cambio, cuando es promovida desde otra área, me piden la colaboración para participar en dicha propuesta a través de apoyo en las actividades o de proporcionar tiempo y espacio". Es debido a esto que, entre más áreas puedan participar de la propuesta, mayor será el grado de aprendizaje que puedan adquirir los estudiantes, y quizás, el objetivo por el cual los líderes de las propuestas innovadoras deben incentivar a colegas y directivos para que realicen su aporte, bien sea incluyendo temáticas relacionadas o proponiendo mejorías en el desarrollo de actividades en cuanto al tiempo, monitoreo y evaluación de la actividad.

Durante las observaciones se pudo apreciar que los docentes JB, MPM y MSD, quienes aplicaron la metodología de ABP (Aprendizaje Basado en Proyectos), describieron el propósito principal del proyecto y la meta principal que había que cumplir para el final del proyecto, donde en algunos proyectos (ciencias sociales) se presentaba una pregunta problema, y en otros (español y tecnología) se presentó una temática específica. La estudiante EH6 comentaba al respecto que "los docentes nos informan sobre el motivo por el cual haré el proyecto, y primero realizan un tipo de introducción, dando a conocer el tema y los objetivos principales que se van a cumplir". Es por esto que, la innovación educativa es vista desde muchos ángulos complementarios, pero lo más importante es que, ante los cambios masivos que se dan en el ambiente educativo, los docentes puedan preservar ese deseo de innovar en su labor para mejorar el aprendizaje de sus estudiantes (García-Peñalvo, 2015). 
Los proyectos pueden variar en su duración y pueden desarrollarse en dos días, o bien extenderse a lo largo del semestre, con el propósito de obtener mejores resultados y mejor aprovechamiento del tiempo por parte de las estudiantes para realizar un producto de buena calidad. El proyecto realizado por JB tomó aproximadamente dos meses y medio, tiempo en que las estudiantes pudieron trabajar con toda tranquilidad, y teniendo todas las instrucciones y actividades a desarrollar publicadas en el campus virtual de la institución.

Después de efectuado el proyecto, se pudo observar que las estudiantes vincularon los contenidos escolares con situaciones de la vida cotidiana, muy relacionadas con su entorno. En el caso de los proyectos vistos en Sociales y Español, involucraron el planteamiento de situaciones de la vida real, como son el cómo organizar un viaje a través del mundo, y cuál es el atractivo más valioso de Colombia dentro de sus departamentos; al momento de mostrar sus productos finales, tuvieron la facultad de explicarlo y asociarlo con el contexto de la realidad en la que viven.

Y es que, cuando se realizan estos proyectos, el docente busca en el estudiante que desarrolle habilidades de colaboración para construir conocimiento; por esto, el aprendizaje colaborativo se convierte en una buena metodología que permite a los estudiantes compartir ideas entre ellos, expresar sus propias opiniones y negociar soluciones, habilidades todas, necesarias en los futuros puestos de trabajo. En el proyecto realizado en el área de Tecnología, las estudiantes tuvieron la oportunidad de realizar el trabajo final inicialmente en documentos separados, donde cada una de las integrantes realizaba sus aportes de acuerdo a la longitud y magnitud del trabajo, y al final, integraban todo en un único trabajo a entregar al docente, $y$, al momento de entregarlo, se pudo ver que las estudiantes demostraron buenas habilidades para la búsqueda de información en internet, para realizar consultas en sitios externos, la elaboración de material audiovisual (videos), la capacidad de decorar interiores con un límite de recursos disponibles, el desarrollo de habilidades de redacción, y desarrollar las habilidades de trabajo en equipo cuando sus integrantes son elegidos por el docente.

Si revisamos los comentarios de algunas de las estudiantes, podremos observar que, los aspectos para la evaluación son conocidos por las estudiantes, y eso se refleja en las diversas expresiones que manifestaron durante cada una de las propuestas de innovación, "se nos evalúa la creatividad, la información, tú manera de trabajar, tú actitud, tú participación en el grupo si se está siendo 
realizado por más personas, el trabajo final"; "se evalúa en creatividad, en cuanto te sepas el tema, en presentación. Y depende si es en parejas o individual que se evalúa el trabajo en equipo"; "se evalúa la creatividad, el uso apropiado del internet, como investigamos y la información, como aprovechamos los recursos que nos da el colegio".

Todos estos comentarios, nos muestran que el factor de creatividad, el uso racional de TIC y el trabajo en equipo en el proyecto son los ítems más relevantes dentro de la institución al momento de ejecutar una propuesta innovadora, y es que, estas tres habilidades son las más complicadas para desarrollar, y las que quizás dan una muestra clara de lo que las estudiantes han aprendido a lo largo del trabajo realizado.

\section{Conclusiones}

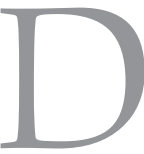

e acuerdo a los resultados obtenidos y partiendo de las tres dimensiones en la que se dividió este proyecto: Factores que limitan la innovación, aprendizaje basado en proyectos y sistematización de la innovación, se describen a continuación los hallazgos más significativos de esta investigación.

La primera división corresponde a los factores que dan ciertas restricciones a las innovaciones aplicadas en el aula, donde se pudo observar y resolver una de las preguntas propuestas de la investigación respecto a las condiciones o factores que limitan la aplicación de las innovaciones en el aula. Se encontró que en la institución, los factores más relevantes son: el tiempo, la motivación de los estudiantes y la falta de participación de los docentes en las propuestas innovadoras.

En el análisis de datos, se encontró que, el motivar a sus estudiantes, y tener el apoyo y participación de sus colegas, puede dar mejores resultados a la propuesta innovadora en el desarrollo de las metas trazadas de la actividad. Y es que, cuando estos dos componentes se juntan (motivación al estudiante y participación docente), se hace más fácil de realizar, porque la propuesta permitirá a los estudiantes ser agentes activos y estar integrados dentro de las actividades propuestas, a su vez en acompañamiento de los docentes, quienes deben actuar como guías antes, durante y después del proceso.

Adicional a esto, se registró cierta ausencia de acompañamiento de parte de los docentes de la institución para apoyar las propuestas realizadas por 
los docentes participantes. En muchos casos se observó que los profesores trabajaron solos y carecieron del apoyo pedagógico de otros profesores. Es importante recordar que las acciones innovadoras demandan organización y acompañamiento de parte de los profesores hacia los estudiantes durante el desarrollo de estas, y que otros docentes pueden dar una mano para apoyar a los estudiantes el desarrollo de las actividades.

Por otro lado, se puso en evidencia que el tiempo es un factor importante para los docentes al momento de implementar una innovación. El tiempo es un elemento importante para la ejecución de las propuestas, y es el que les da el espacio para ejecutar estas propuestas, para poder obtener los mejores resultados y se puedan llegar a institucionalizar.

Otro de los hallazgos, es el que se refiere a la aplicación del Aprendizaje Basado en Proyectos, la cual es una de las metodologías promovidas por la institución para generar el aprendizaje significativo y el trabajo colaborativo entre las estudiantes. En general, en las observaciones de clase y en las planeaciones curriculares, se observó que las estudiantes desarrollan un proyecto, proponen un plan con estrategias definidas, y concluyen con una solución a un interrogante o una situación problema. Estas características, fueron evidentes en las observaciones de clases, donde se apreció la forma cómo el docente hacía un preámbulo de lo que sería el proyecto y de cuál sería la pregunta o situación problema, de cómo organizaba a las estudiantes para realizar este trabajo, de forma que cada una de ellas tuviera un rol que asumir para iniciar el desarrollo del producto, y el modo en el que las evaluaría, bajo el uso de rúbricas o de ciertos parámetros dados en la clase.

Un hallazgo relevante dentro de la investigación fue la falta de motivación de parte de las estudiantes para ejecutar estas actividades. El observar que las estudiantes, en ciertos momentos no tomaban en serio o no mostraban el interés deseado, reflejaba que la actividad no llevaba la motivación suficiente. Esto es, porque el grado de responsabilidad dentro de la innovación educativa es mutuo y conlleva a tener un alto grado de compromiso de los involucrados en estas propuestas (alumnos, docentes, directivos). Por ende, comprometiendo a la comunidad docente en el apoyo de las actividades a desarrollar, integrando áreas de conocimiento transversales a la propuesta, y demostrando a los estudiantes que el acompañamiento se dará de esta manera se puede garantizar el éxito que se desea: un aprendizaje significativo y más motivación para el mejoramiento del aprendizaje de los estudiantes en el aula, significaría una mejora en la calidad pedagógica para la institución educativa. 
Es importante señalar que las estudiantes realizan proyectos de manera frecuente, y esto puede influir en el desempeño y el interés de ellas para con las propuestas; dado esto, realizar un análisis exhaustivo de cómo las estudiantes han trabajado en las últimas propuestas realizadas, y con qué frecuencia han trabajado en dicha modalidad por proyectos, puede ser útil para tener en cuenta qué cambios pueden ser implementados y que sean de mejor provecho para la parte motivacional, organizacional y académica de las estudiantes.

Otra de las conclusiones de esta investigación es que las innovaciones deben ser reguladas y retroalimentadas por los docentes y las directivas, y dar un espacio relevante a los estudiantes para que puedan opinar sobre estas. Para esto, es importante que, al momento de realizar la evaluación, se generen indicadores relacionados con la innovación aplicada, que les permitan identificar aspectos relevantes de dicha propuesta (creatividad, manejo del tiempo, utilización de recursos), que puedan determinar si la propuesta puede ser aplicada una vez más o si es preferible que se cambie o modifique.

En la planeación de actividades, deben considerar el factor del tiempo para la realización de las actividades. Los docentes deben tener presentes las variaciones que pueden haber en las actividades escolares en cuanto al horario escolar que manejan, la duración de la clase y el calendario escolar de la institución; por ende, es clave no apresurar el desarrollo de actividades y organizar junto con las estudiantes para el buen manejo del tiempo tanto dentro como fuera del aula; contar siempre con un plan alternativo, que implique la participación de otros docentes, dado que pueden surgir inconvenientes para la aplicación de la actividad, tales como las incapacidades por cuestiones de salud o la disponibilidad horaria del docente; realizar investigación sobre las acciones o metodologías implementadas, para detectar aspectos claves para el mejoramiento y la aplicación de las propuestas o bien para sugerir alternativas e innovaciones.

Con base a lo descrito, se planearon consideraciones reflejadas en esta investigación que abren interrogantes para dar apertura a otras investigaciones, en las cuales se pueden tener en cuenta:

- Indagar sobre los apoyos que brindan los directivos a los docentes, que va desde la capacitación, incentivos, políticas para implementar innovaciones, adquisición de recursos, vinculación con la academia, padres de familia y alumnos. 
- Evaluar el impacto de las TIC como herramienta que potencia el aprendizaje y de la metodología del aprendizaje basado en proyectos. En particular indagar sobre la adquisición de habilidades y competencias de los estudiantes que desarrollan proyectos y usan herramientas tecnológicas.

- Indagar sobre los diferentes tipos de capacitaciones que reciben los docentes, el aprendizaje que obtienen y la aplicación que llevan a cabo en el aula.

\section{BIBLIOGRAFÍA}

Carbonell, J. (2002). La aventura de innovar. El cambio en la escuela. España: Editorial Morata.

Díaz, M.; Díez, J.; San Fabián, J. \& Martínez, P. (1996). Innovación educativa y desarrollo profesional docente. En M. Díaz, El desarrollo profesional docente y las resistencias a la innovación educativa (págs. 15-39). Oviedo: Universidad de Oviedo.

García-Peñalvo, F. J. (2015). Mapa de tendencias en innovación educativa. Teoría de la educación. educación y cultura en la sociedad de la información, 16() 6-23. Recuperado de http://www.redalyc. org/articulo.oa?id=201043053001

Hernández, J. \& Castañeda, E. (2001). Profesión docente. Revista Iberoamericana de Educación (25).

Marcelo, C. (2013). Las tecnologías para la innovación y la práctica docente. Revista Brasileira de Educação, 18() 25-47. Recuperado de http://www.redalyc.org/articulo.oa?id=27525615003

Ministerio de Educación de España. (2011). Estudio sobre la innovación educativa en España. Madrid: Centro de Publicaciones.

Pintrich, P. (1994). Student motivation in the college classroom. Estados Unidos: Greenwood Press.

Polanco, A. (2005). La motivación en los estudiantes universitarios. Actualidades Investigativas en Educación, 5(2), 1-13.

Rimari, W. (2003). Para sistematizar experiencias. Innovando (20).

Ríos, D. (2003). Factores y obstaculizadores de la innovación educativa. Revista de Estudios y Experiencias en Educación, 3(2).

Ríos, D. (2010). Factores que inciden en el clima de aula universitario. Revista Latinoamericana de Estudios Educativos, vol. XL, $3^{\circ}$ y $4^{\circ}$ trimestres, México, D.F., pp. 105-126. Latindex, Redalyc. Valenzuela, J., \& Flores, M. (2011). Fundamentos de investigación educativa. México, D.F.: Editorial TecMonterrey.

Villa Lever, L. (2014). Educación media superior, jóvenes y desigualdad de oportunidades. Innovación educativa, 14() 33-45. Recuperado de http://www.redalyc.org/articulo.oa?id=179430480004 

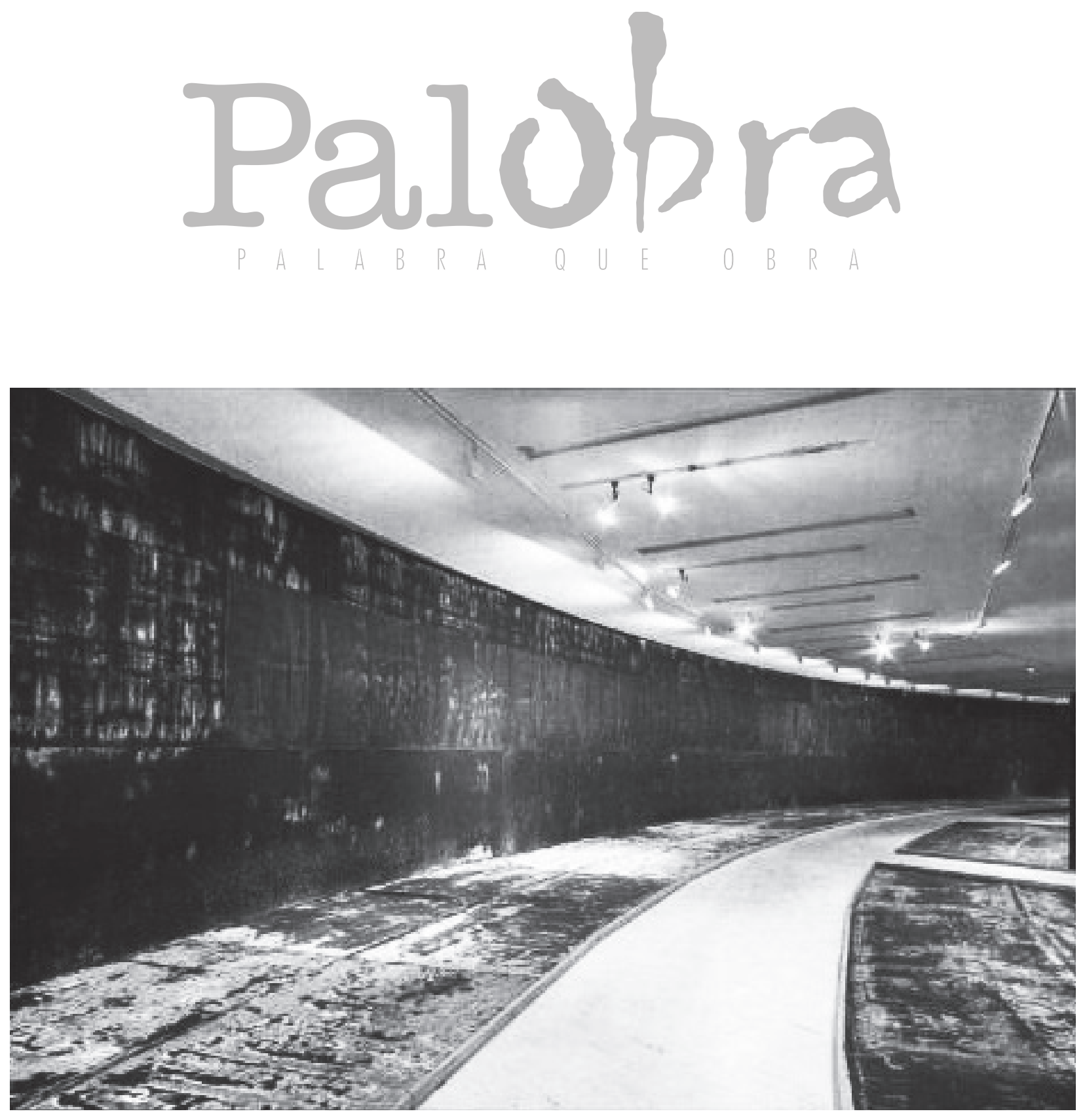

Un bosque, un jardín. 1997. Instalación. Mención de honor en el primer Premio Luis Caballero. 